\title{
Fuzzy Comprehensive Evaluation System Based on the Matter-Element Model
}

\author{
Bo Zhang ${ }^{1, a}$, Wei Gao ${ }^{2, b}$ and Xiaowei Han ${ }^{3, c}$ \\ 1Shenyang University, School of Information Engineering, Shenyang, China \\ 2Shenyang University, School of Information Engineering, Shenyang, China \\ ${ }^{3}$ Shenyang University, School of Information Engineering, Shenyang, China \\ aprogramboy007@163.com, b1010930035@qq.com, chxw69@163.com
}

Keywords: fuzzy comprehensive evaluation, matter-element model, talent evaluation.

Abstract. In order to improve the ability of fuzzy comprehensive evaluation of talents, this paper proposes a fuzzy comprehensive evaluation method which is the combination of qualitative and quantitative. Results show that the system can ensure the accuracy of the evaluation results, can meet the need of the fuzzy comprehensive personnel evaluation, and can provide technical support for related departments on talent evaluation rapidly and accurately.

\section{Introduction}

Diversity of talent research capacity requires that scientific research talent management should be more humanized, scientific and adaptability [1]. At present, ways of domestic talent work are old, talent management mechanisms are lagging and talent attraction is not enough. To solve these problems researches mainly depend on the work experience and the performance inference method with the traditional human resource theory [2], which lacking of scientific and effective information technology as support and cannot be adapted to the increase of the number of the talented person and the type of diversification. It is difficult to meet the requirements of talents to work in the new period.

The extenics founded by Chinese scholars Cai in 1983 adopts formalized tools to study rules and methods to solve the problem of contradiction from the two perspective of qualitative and quantitative [3]. Basis of extenics are the matter-element theory and extension set theory, and the logic cell is matter-element. To solve the problem of fuzzy comprehensive evaluation of talents, the matter-element and extension set can compute the probability that a talent belonging to a degree according to the attribute quantity of the talent, and the correlation function can make diagnosis refinement and quantitative. So it can provide a new way to solve the problem of talent fuzzy comprehensive evaluation from the angle of the change.By summarizing existing research results, this paper firstly establishes the talent information matter-element model, which is mainly supported by the matter-element theory; secondly, this paper proposed a novel way of fuzzy comprehensive evaluation of the talent information with the evaluation calculation of talent belonging to a certain extent by the introduction of correlation function.

\section{Related Work}

Scientific and technological personnel evaluation work involves many sectors and essential factors. According to different evaluation objects and contents, there are a variety of evaluation principles, evaluation ideas, evaluation indexes and evaluation methods of choice. The fundamentals of scientific and technological personnel evaluation used at home and abroad including competency model theory, the principle of individual difference, the thought of fuzzy principle, the measurability of things consistent principle, evaluation principle and so on. The behavioral science, psychology, management science, metrology and personnel measure theory are also used as the theoretical basis for scientific and technological personnel evaluation [4]. The matter-element model has the characteristics of fuzziness and diversity, which are more suitable to solve the problem of incompatibility among the contestant factors in the process of the multiple factors and multi-criteria decision-making. Therefore, the model has been widely used in such fields like the ecological 
environment [1, 2], the urban power grid planning, land consolidation [3], disaster prediction [4], water resources evaluation [5], low carbon logistics [6], and so on in recent years. Instances show that the method in the multi-index comprehensive evaluation can get a better evaluation result.

\section{Fuzzy comprehensive evaluation model based on the matter-element model}

Matter-element model proposed by Cai study the rules and methods to solve the problem of contradiction from the perspective of qualitative and quantitative by the formal tool. For solving the fuzzy comprehensive evaluation of talents, the matter-element model and extension set can evaluate the ability of talent according to various features of the talent value. At the same time, correlation functions can make diagnosis refined and quantitative, which providing a new way to fuzzily and comprehensively evaluate talents from the angle of the change.

Model Definition. In the talent evaluation process, as a talent often has multiple attribute values in an attribute dimension so that the evaluation process is suitable for matter-element model to describe.

Definition 1. The talent fuzzy comprehensive evaluation model based on matter-element model: evaluation system model is a set of evaluation criteria, denoted as $\mathrm{N}, \mathrm{N}=<\mathrm{N}_{1}, \mathrm{~N}_{2}, \ldots, \mathrm{N}_{\mathrm{M}}>$. The $\mathrm{N}_{\mathrm{j}}$ is the $\mathrm{j}$-th evaluation criteria, which including the classical domain and the joint domain.

The classic form of the $\mathrm{N}_{\mathrm{j}}$ domain are described as follows:

$$
R_{j}=\left[\begin{array}{c}
n_{j} c_{1}<a_{1}, b_{1}> \\
c_{2}<a_{2}, b_{2}> \\
c_{3}<a_{3}, b_{3}> \\
\cdots \cdots \\
c_{m}<a_{m}, b_{m}>
\end{array}\right]
$$

Among them, $a_{i}$ and $b_{i}$ represent the common range of attribute $c_{j}$. Section of domain $\mathrm{N}_{j}$ is described as follows:

$$
R_{p j}=\left[\begin{array}{cc}
n_{j} c_{1} & <a_{p 1}, b_{p 1}> \\
c_{2} & <a_{p 2}, b_{p 2}> \\
c_{3} & <a_{p 3}, b_{p 3}> \\
\cdots & \ldots \\
c_{m} & <a_{p m}, b_{p m}>
\end{array}\right]
$$

Among them, $a_{\mathrm{pi}}$ and $\mathrm{b}_{\mathrm{pi}}$ represent the possible value range of the $\mathrm{c}_{\mathrm{i}}$ property.

Talent Evaluation Process based on Matter-Element Model. The matter-element model firstly divides the evaluation of the object level into several grades according to the expert experience and advice, and secondly calculates the correlation of each evaluation index belonging to various levels, and thirdly compares the evaluation results with correlation degree of each level, and selects the level having the maximum correlation degree as the final results of the evaluation at last. The bigger the correlation degree is, the higher the set of the grade of the degree of compliance is.

The basic process of the matter-element model fuzzily and comprehensively evaluating the information of the talents is as follow:

1. Constructing the matter element representation of talent information;

2. Constructing the rule set and the corresponding classical domain fields and section fields of an evaluation model;

3. Calculating the correlation between the talent information and each evaluation standard; 
4. Calculating the weight coefficient of correlation;

5. Rating calculation.

In view of the above basic process, detailed steps of fuzzy comprehensive evaluation on the personnel information based on the matter-element model are as follows:

Step 1: Constructing the matter element representation of talent information. For the evaluation of scientific and technological personnel, it is needed to describe multiple attributes of a talent, such as personal basic information, published articles and books, participation in the project, awards and honorary titles. Such a complex data structure is suitable for the matter element model.

Step 2: Constructing the rule set and the corresponding classical domain fields and section fields of an evaluation model. For different departments having inconsistent talent evaluation criteria, single standard of talent evaluation cannot be used for different departments. In the evaluation system of this paper, the user can customize the evaluation target, and set the classical domain and joint domain corresponding to each evaluation target. Thus, while the user is defining the evaluation system of $\mathrm{N}$, it is needed to specify the corresponding evaluation criteria set, which is $\mathrm{N}=<\mathrm{N}_{1}, \mathrm{~N}_{2}, \ldots$ $\mathrm{N}_{\mathrm{m}}>$. At the same time, users need to specifies the range of commonly used values $<\mathrm{a}_{\mathrm{j}, \mathrm{k}}, \mathrm{b}_{\mathrm{j}, \mathrm{k}}>$ and available range of values for $<\mathrm{a}_{\mathrm{pk}}, \mathrm{b}_{\mathrm{pk}}>$ for each evaluation item $\mathrm{c}_{\mathrm{j}, \mathrm{k}}$ of $\mathrm{N}_{\mathrm{j}}$. So the system can be based on user specified values to generate the classic $\mathrm{N}$ domain $\mathrm{R}_{\mathrm{j}}$ and domain $\mathrm{R}_{\mathrm{pj}}$.

Step 3: Calculating the correlation between the talent information and each evaluation standard. In the process of personnel fuzzy comprehensive evaluation based on matter element model, the correlation degree gives the possibility of the talent information to meet the evaluation standard $\mathrm{N}_{\mathrm{j}}$.

Among them, $\mathrm{k}_{\mathrm{j}}\left(\mathrm{t}_{\mathrm{i}}\right)$ means correlation between talent $\mathrm{i}$ information and the $\mathrm{j}$-th evaluation criteria, $\mathrm{d}\left(\mathrm{v}_{\mathrm{i}}, \mathrm{v}_{\mathrm{j}, \mathrm{i}}\right)$ means the $\mathrm{i}$-th index of matter element value with the classical domain distance, $\left|\mathrm{v}_{\mathrm{j}, \mathrm{i}}\right|$ means the number of values in $\mathrm{N}_{\mathrm{j}}$, the $\mathrm{d}\left(\mathrm{v}_{\mathrm{i}}, \mathrm{v}_{\mathrm{pj}}, \mathrm{i}\right)$ means the $\mathrm{i}$-th index element value and joint domain of distance.

Step 4: Calculating the weight coefficient of correlation. The weight coefficient of correlation degree is used to indicate the importance of the value of each matter element in the evaluation process. In this paper, the entropy model is used to calculate the weight coefficient of correlation degree. Entropy describes the degree of disorder within a system. Assuming there are events within the system like $S_{1}, S_{2}, \ldots S_{n}$. Then, the more the number of events within the system, the greater the entropy of the system is. In this paper, the properties of the attribute value distribution are used to quantify the role of attribute in the process of fuzzy comprehensive evaluation of talents. If the width of the attribute value distribution is larger, the higher the talent difference is, and the higher the attribute description is. At this point, based on the attribute of the talent evaluation it can increase the difference of evaluation results, so as to avoid the phenomenon of convergence of the evaluation results.

Due to different department for talent evaluation itself has its own requirements, therefore, the weight $\mathrm{w}_{\mathrm{i}}$ is calculated by the combination of artificial set and attribute entropy in this paper.

Step 5: Rating calculation. Value of the correlation function $\mathrm{K}(\mathrm{t})$ evaluates the degree of the unit in accordance with a standard range of membership. $\mathrm{K}(\mathrm{t}) \geq 1.0$ means the one being evaluated has exceeded the maximum limit standard. The larger the value is, the greater the development potential is. When $0 \leq \mathrm{K}(\mathrm{t}) \leq 1.0$, it means the degree of the one being evaluated conforms to the requirements of the standard. The greater the value is, the closer it is to the standard limit. When $-1.0 \leq \mathrm{K}(\mathrm{t}) \leq 1.0$, it means the one being evaluated dose not conform to the requirements of the standard, but has the possibility of being transformed into standard object conditions. The greater the value is, the easier the transformation will be. When $\mathrm{K}(\mathrm{t}) \leq-1.0$, it means the one being evaluated do not conform to the requirements of the standard, and does not have opportunity to be converted to standard conditions.

\section{Design and implementation of personnel fuzzy comprehensive evaluation system based on matter element model}

The overall business process of the talent evaluation software based on the fuzzy matter element model includes two main steps: First is designing evaluation criteria by user according to the actual need, and then modeling evaluation standard by the system and evaluating the personnel information. 
The system integrates professional assessment evaluation technology, and uses three layer $\mathrm{C} / \mathrm{S}$ (client/ server) structures. The presentation layer, which containing the external users display interface, mainly realizes man-machine interactive graphic interface design, and realizes the basic application interface and professional evaluation. The middle layer, which containing the business logic layer, correlates data of talents and data attributes together, and responsible for the attribute editor, management, searching, accounting, and implements the algorithm based on matter-element evaluation model. The data layer, which being implemented by SQL Server 2008, is responsible for personnel information andthe storage and management of evaluation index.

The system uses the information of high-level person in shenyang as the research object. Based on the dot net Framework 4.5 framework, it uses the Visual Studio 2013 as the integrated development environment, uses SQL Server 2008 as the database. The system mainly implements the basic function like construction of talent evaluation rule, construction and management of talent assessment information. And it also realizes the model and algorithm for fuzzy comprehensive evaluation of personnel information.

\section{Conclusions}

This paper fuzzily and comprehensively evaluates the talent information based on the matter-element model, which having advantages of solving incompatible problem. Software uses Visual Studio 2013 and SQL Server 2008 database to build a system for talent information fuzzy comprehensive evaluation. From the result of implementing the software into the high-level personnel information evaluation in Shenyang, the software performs will, and shows a broad application prospect in the field of talent fuzzy comprehensive evaluation.

\section{Acknowledgements}

This work was financially supported by the Shenyang Science and Technology Project (F14 - $230-5$ - 34).

\section{References}

[1] Hui Dai, Yueming Hu, Jianwen Xie, et al. Suitable Evaluation System of Camellia Planting based on GIS and Matter-Element Model [J]. COMPUTER ENGINEERING AND DESIGN. 2014, 35(3), pp:1100-1104.

[2] Shijun Tang, Rajan Alex: Synthetic Rating on Talent Evaluation-Similarity of Subsets. Complex Adaptive Systems 2015:367-372

[3] Ang Li: College Talents Combination Evaluation and its Empirical Study. WHICEB 2013:47

[4] Gol Kim, Yunchol Jong, Sifeng Liu, Choe Rim Shong: Hybrid Grey Interval Relation

Decision-Making in Artistic Talent Evaluation of Player CoRR abs/1207.3855 (2012)

[5] ZHAN Guohui, QI Yan, TAN Xianlin, CAO Jia, ZHANG Yi. Classification Method of Urban

Public Traffic Hub Station Based on Matter Element Analysis and Statistical Analysis. Journal of Chongqing Jiaotong University:Natural Science. 2016, 3. pp:147-156

[6] Shijun Tang, Rajan Alex:Synthetic Rating on Talent Evaluation-Similarity of Subsets. Complex Adaptive Systems 2015: 367-372 